\title{
IMPROVING MARKOV RANDOM FIELD BASED SUPER RESOLUTION MAPPING THROUGH FUZZY PARAMETER INTEGRATION
}

\author{
D. R.Welikanna ${ }^{\text {a, }}$, M. Tamura ${ }^{\text {a }}$, V. A. Tolpekin ${ }^{\text {b }}$, J. Susaki ${ }^{\text {a }}$, M. Maki ${ }^{\text {a }}$ \\ ${ }^{a}$ Dept. of Civil and Earth Resources Engineering, Kyoto University, 615-8540 Kyoto, Japan - (tamuram@envinfo.uee, \\ d.welikanna@hx7.ecs, susaki@envinfo.uee, maki@envinfo.uee).kyoto-u.ac.jp \\ ${ }^{\mathrm{b}}$ Faculty of Geo-Information Science and Earth Observation(ITC), University of Twente, P.O.Box 217, 7500AE \\ Enschede, The Netherlands- tolpekin@itc.nl
}

Commission VII, WG VII/4

KEY WORDS: Vague Urban Land-cover, SAM, MRF, SRM, Fuzzy mean and Fuzzy covariance

\begin{abstract}
:
The objective of this study was to improve the Markov Random Field (MRF) based Super Resolution Mapping (SRM) technique to account for the vague land-cover interpretations (class mixture and the intermediate conditions) in an urban area. The algorithm has been improved to integrate the fuzzy mean and fuzzy covariance measurements, to a MRF based SRM scheme to optimize the classification results. The technique was tested on a WORLDVIEW-2 data set, acquired over a highway construction area, in Colombo, Sri Lanka. Based on the visual interpretation of the image, three major land-cover types of this area were identified for the study; those were vegetation, soil and exposed grass and impervious surface with low medium and high albedo. The membership values for each pixel were determined from training samples through Spectral Angle Mapper (SAM) technique. The compulsory fuzzy mean and the covariance measurements were derived using these membership grades, and subsequently was applied in MRF based SRM technique. The primary reference data was generated using Maximum Likelihood Classification (MLC) performed on the same data which was resampled to $1 \mathrm{~m}$ resolution. The scale factor was set to be $(S)=2$, to generate $S R M$ of $1 \mathrm{~m}$ resolution. The smoothening parameter $(\lambda)$ which balances the prior and likelihood energy terms were tested in the range from 0.3 to 0.9 . SRM were generated using fuzzy MRF and the conventional MRF models respectively. Results suggest that the fuzzy integrated model has improved the results with an overall accuracy of $85.60 \%$ and kappa value of 0.78 between the optimal results and the reference data, while in the conventional case it was $77.81 \%$ of overall accuracy with kappa being 0.65 . Among the two MRF models, fuzzy parameter integrated model shows the highest agreement with class fractions from the reference image with a smallest average _MAE (MAE, Mean Absolute Error) of 0.03.
\end{abstract}

\section{INTRODUCTION}

Pattern recognition using satellite imagery has been challenged by the urban land scape in establishing a precise relationship between the pattern and a class label. Primarily the rapid change of the urban land-cover categories within a small distance resulting similar entities at different locations, majority of landcover types being internally heterogeneous and intermediate conditions of the class boundaries make the urban landscape a vague entity (Wang, 1990; Wood and Foody, 1993). This situation on the ground makes a mix spectral signature within a pixel of a satellite image (Zhang and Foody, 2001). Still the classes are mutually exclusive and discrete in the ground it might not be the case in the image due to the sensor spatial resolution. In many cases this brings fuzziness or the uncertainty into the image in the form of mixed pixels (Fisher et al., 2006). Fuzzy set theories have been used extensively to address this imprecise class information in a mixel (pixel comprising mixed land cover categories) which complicate the classification problem (Zadeh, 1965). Although it should be noted that fuzzy parameter integrated classification schemes does not fully resolves the problem of class mixtures within the pixel, it provides more appropriate definition for vague landcover classes recorded at a particular sensor resolution. MRF have been potentially identified in Remote Sensing image classification with promising results, mainly due to its ability to integrate the contextual based information in to the classification scheme (Kasetkasem et al., 2005; Solberg et al., 1996). This practical applicability of MRF has been made possible by the equivalence between MRF and Gibbs distribution, established by the Hammersley-Clifford theorem (Li, 2009; Tso and Mather, 2009). This provides a convenient framework to determine the joint prior probability for the pixel labelling problem. One of the main applications of the MRF is to produce higher resolution land-cover maps also called SRM from coarser resolution satellite images (Kasetkasem et al., 2005; Welikanna et al., 2008). Moreover MRF models have been widely used to resolve many of the vision problems including image restoration and segmentation, edge detection, texture analysis, data-fusion and change detection (Ardila et al., 2011; Kasetkasem and Varshney, 2002; Xu et al., 2011). MRF based SRM techniques have been using class descriptive statistics in the form of conventional class mean and covariance to model class probability density functions. The conventional mean and the covariance, consider a training pixel to belong to a single class (one pixel one class) while neglecting its proportional contribution. As an extension to this conventional scheme, several previous studies have integrated fuzzy class parameters reliably into a classification scheme (Wang, 1990; Zhang and Foody, 2001, Tang et al.,2007). In this study we propose a robust MRF model which integrates the fuzzy class descriptive statistics, to produce SRM with improved classification results. 


\section{MRF AND THE FUZZY PARAMETERS}

This section mainly focuses on illustrating the key conceptual elements of the fuzzy parameter integrated MRF model.

\subsection{MRF and images}

In any real image adjacent pixels are correlated due to the point spread function (PSF) effect and the ground cover types being well distribute over regions lager than the spatial resolution of the image (Richards and Jia, 2006). There for with the use of the contextual information, pixels will no longer be treated as an isolated entity. MRF with its relationship to the Gibbs Random Fields (GRF), layout the framework needed to model this contextual dependency systematically. A comprehensive introduction to this can be found in the literature as well $(\mathrm{Li}$, 2009; Tso and Mather, 2009). Let a set of pixel DN values in an observed image $\mathbf{x}$ with $K$ spectral bands, are represented by $x_{1} \ldots x_{m}$, where $M$ is the total number of pixels to be classified. The measurement vectors are represented as $x_{m}: m=1, \ldots M$, in a pixel matrix $A \in\left(M_{1} \times M_{2}\right)$. Let $\mathbf{c}$ be the resulting SRM defined on a pixels matrix $B$, with each of the pixel belonging to a unique class at a finer resolution than the observed image. Let an unobserved multispectral image be $\mathbf{y}$ with the same number of spectral bands as $\mathbf{x}$ but with a spatial resolution identical to $\mathbf{c}$. The pixel locations in $\mathbf{y}$ are represented by $y_{i, j}$. Also it is assumed that each pixel in y can be assigned to a unique class $\left(c\left(y_{i, j}\right)\right)$. If the pixel resolution of the original image is $R$ and that of the SRM is $r(<R)$, the relation between the two images is determined by a scale factor which is denoted by $S=R / r$. Hence it can be seen that the pixel matrix $B=S M_{1} \times S M_{2}$. In this study $S$ takes an integer value for the convenience. For each pixel $y_{i, j}$ a symmetric neighbourhood $N\left(y_{i, j}\right)$ is defined by a window size $W$, where it is the length of the side of the squared window. Different definitions for the neighbourhood system can be found in the literature $(\mathrm{Li}, 2009)$. A second order neighbourhood consisting the eight closest connected pixels $(W=3)$ have been selected for this study. The classified image c will be modelled as a MRF with respect to the Neighbourhood system $N\left(y_{i, j}\right)$. MRF is defined by local properties, therefore the labelling of the pixels is considered to be effected by this neighbourhood configuration (Tso and Mather, 2009). This is where the MRF models take the advantage of modelling contextual dependencies, or the spatial correlation among the pixels. The set $\omega$ is referred as a random field with the probability distribution $p(\omega)$ describing the likelihood of finding the labels $c\left(y_{i, j}\right)=\omega \in\{1, \ldots l\}$ over the image. We model the classified image $\mathbf{c}$ as a MRF with the neighbourhood system $N\left(y_{i, j}\right)$. The overall objective of the model is to classify all the pixels which maximize the global posterior probability $p(\omega \mid \mathbf{x})$, which is the probability that $\omega$ is the correct overall scene labelling given the full set of measurement vectors $\mathbf{x}$. According to the Bayes theorem a pixel $x_{m}$ in the observed image is allocated to a class $\omega$ according to Eq. (1), where a value $\omega$ is selected to maximize the argument for a pixel to find the most appropriate scene label $\bar{\omega}$.

$$
\bar{\omega}=\underset{\omega}{\arg \max }\{p(\mathbf{x} \mid \omega) p(\omega)\}
$$

According to the equivalence of the MRF and the GRF the probabilities in Eq. (1) are defined by the means of energy functions. GRF describes the global properties of an image, where a label given to a pixel is affected by the label given to all the other pixels, according to the joint distribution of classes for all the pixels. Hammersley-Clifford theorem explains the existence of a unique GRF for every MRF as long as the GRF is defined by the cliques on a neighbourhood system. A clique is a subset in which all pairs of the pixels are mutual neighbours (Tso and Mather, 2009). Hence the prior probability $P(\omega)$ for the SRM, the conditional probability $p(\mathbf{x} \mid \omega)$, that the image $\mathbf{x}$ is observed given the true SRM and the posterior probability $p(\omega \mid \mathbf{x})$ are modelled by means of energy functions. Owing to this relation the posterior probability in Eq. (1) also takes the form given by,

$$
p(\omega \mid \mathbf{x})=\frac{1}{Z} \exp (-U(\omega \mid \mathbf{x}) / T)
$$

Where $Z$ is the normalizing constant, $T$ is the constant termed temperature and $U(\omega \mid \mathbf{x})$ is the posterior energy function of the super resolution map $\mathbf{c}$ given the observed image $\mathbf{x}$. Both the terms $Z$ and $T$ are independent of $\omega$ and $\mathbf{x}$. Based on Eq. (2) it can be shown that maximizing the $p(\omega \mid \mathbf{x})$ is equivalent to minimizing the energy function $U(\omega \mid \mathbf{x})$.

\subsection{Prior Energy to model the contextual dependencies}

In the MRF, prior and likelihood energies are modelled individually. They account for the contextual and spectral properties of the observed image respectively. The prior energy is modelled by using the sum of pair-site interaction within the neighbourhood system $\left(N\left(y_{i, j}\right)\right)$, as follows:

$$
\begin{gathered}
U(\omega)=\sum_{i, j} U\left(c\left(y_{i, j}\right)\right) \\
=\sum_{i, j} \sum_{l \in N\left(y_{i, j}\right)} \beta\left[1-\delta\left(c\left(y_{i, j}\right), c\left(y_{l}\right)\right)\right]
\end{gathered}
$$

In Eq. (3) each pixel contributes locally to the prior energy, which is denoted as $U\left(c\left(y_{i, j}\right)\right)$. The term $\delta\left(c\left(y_{i, j}\right), c\left(y_{l}\right)\right)$ is called the "Kroneker delta", which takes a unit value when $\delta\left(c\left(y_{i, j}\right)=c\left(y_{l}\right)\right)$ and 0 otherwise (Richards and Jia, 2006). $\beta>0$ controls the influence of the neighbouring pixels, and can be an anisotropic or isotropic assumption made by the user. In this study this value is an isotropic expression which only depends on the Euclidian Distance $D$ between the pixels $y_{i, j}$ and $y_{l}$ written as:

$$
\beta=\frac{1}{\varpi} \times\left[D\left(c\left(y_{i, j}, y_{l}\right)\right)\right]^{-2}
$$

Where $\varpi=\sum_{l \in N_{i, j}} D\left(c\left(y_{i, j}, y_{l}\right)=1\right.$ is the normalizing constant. A single pixel in the observed image is considered to resemble a multidimensional normal distribution. This distribution is determined by the mean and the variance of each spectral class. To implement the fuzzy integrated MRF model, these fundamental parameters have been reformed using fuzzy definitions. Subsequently they were used to define the likelihood energy. 


\subsection{FUZZY mean and FUZZY covariance}

Fuzzy set theory (Zadeh, 1965) provides the conceptual framework to solve the classification problems in an ambiguous environment. In the problem of image classification if we take an event $\omega$ which is a class label and consider it to be a fuzzy subset of the universe of discourse $\psi$, and $\mathbf{x}$ to be a feature vector of a particular pixel, then the probability density function of $\omega$ can be represented by a fuzzy membership function $f_{\omega}$ as follows

$$
p(\omega)=\int_{\psi} f_{\omega}(\mathbf{x})
$$

This multiple membership values for feature vector realize how much it contributes to a land-cover class label, this change its contribution to the class mean and the variance. Accordingly the discrete fuzzy mean and the fuzzy covariance matrices can be derived from the definitions given by Wang (1990). Here the conventional mean and the covariance will be restructured using fuzzy membership function. These membership values were defined by the class fractions within a pixel of the coarser resolution input image. In the case of per-class covariance matrices, let the training pixels take values $x_{1}, x_{2}, \ldots ., x_{n}$ with $n$ being number of training pixels for an $l$ number of classes. Then the respective fuzzy training data set can be represented as a fuzzy $\operatorname{set}\left\{F_{l \times n} / f_{i, j} \in[0.0,1.0]\right\}$. The element $f_{i, j}$ represent the fuzzy membership value of a training pixel $x_{j}(1 \leq j \leq n)$ to the $l$ class. The discrete fuzzy mean $\overline{\mu_{\omega}}$ and the fuzzy covariance matrix $\bar{\Sigma}_{\omega}$ for class $\omega$ can be defined as follows:

$$
\begin{aligned}
& \overline{\mu_{\omega}}=\frac{\sum_{i=1}^{n} f_{\omega}\left(x_{i}\right) x_{i}}{\sum_{i=1}^{n} f_{\omega}\left(x_{i}\right)} \\
& \overline{\sum_{\omega}}=\frac{\sum_{i=1}^{n} f_{\omega}\left(x_{i}\right)\left(x_{i}-\overline{\mu_{\omega}}\right)\left(x_{i}-\bar{\mu}_{\omega}\right)^{T}}{\sum_{i=1}^{n} f_{\omega}\left(x_{i}\right)}
\end{aligned}
$$

Where $f_{\omega}\left(x_{i}\right)$ is the fuzzy membership value for the pixel vector $x_{i} \equiv x_{m}$ in the observed image $\mathbf{x}$. Parameter estimation was done at the coarser resolution scale. To determine the class membership grades for each pixel different approaches can be found in the literature, for an evaluation additional research is required. In this study we have unmixed the pixel vectors to their respective class compositions to define the membership values for each pixel. A single pixel in the coarser resolution image $\mathbf{x}$, corresponds to $S^{2}$ pixels in the finer resolution SRM, c. Hence the probability density function of an observed pixel vector $x_{m}$ is assumed to be normally distributed with respect to the pixel composition with mean $\bar{\mu}(\mathbf{x})$ and covariance $\bar{\Sigma}(\mathbf{x})$, which can be defined using Eq. (6) as follows:

$$
\begin{aligned}
& \bar{\mu}(\mathbf{x})=\sum_{\omega=1}^{l} \theta_{\omega}(\mathbf{x}) \overline{\mu_{\omega}} \\
& \bar{\Sigma}(\mathbf{x})=\sum_{\omega=1}^{l} \theta_{\omega}(\mathbf{x}) \overline{\sum_{\omega}}
\end{aligned}
$$

Here $\theta_{\omega}$ is the proportion of the class $\omega$ in a pixel $x_{m}$, where $\sum_{\omega=1}^{L} \theta_{\omega}(\mathbf{x})=1$. We also assume the spectral values of
$S^{2}$ fine resolution pixels $y_{i, j}$ are independent and identically distributed, according to the normal distribution of the parameters of class $c\left(y_{i, j}\right)$.

\subsection{Likelihood energy to model the spectral information}

The conditional distribution of the observed data $\mathbf{x}$ with the given true class labels $\omega$, is assumed to be Gaussian. We also assume a coarser spatial resolution pixel of the original image to contain a number of pure pixels at the fine spatial resolution. These fine spatial resolution pixels are strictly assumed to be spatially un-correlated. Therefore the fuzzy mean and the fuzzy covariance matrix of the observed pixel at a coarser resolution scale are directional sum of fuzzy mean vector and the fuzzy covariance matrix of the corresponding pixels at the fine resolution scale. Hence the conditional probability density function (PDF), as shown in Eq. (1) for the observed image can be defined as follows:

$$
P(\mathbf{x} \mid \omega)=\prod_{m} \frac{1}{(2 \pi)^{K / 2}\left|\overline{\sum(\mathbf{x})}\right|^{1 / 2}} \times \exp \left(-\frac{1}{2}\left(x_{m}-\overline{\mu(\mathbf{x})}\right)^{t} \bar{\Sigma}(\mathbf{x})^{-1}\left(x_{m}-\overline{\mu(\mathbf{x})}\right)\right)
$$

The likelihood energy in this case with respect to the relationship shown in Eq. (2) takes the form written by:

$$
U(\mathbf{x} \mid \omega)=\sum_{m, l}\left[\frac{1}{2}\left(x_{m}-\bar{\mu}(\mathbf{x})\right)^{t} \bar{\Sigma}^{-1}(\mathbf{x})\left(x_{m}-\bar{\mu}(\mathbf{x})\right)+\frac{1}{2} \ln |\bar{\Sigma}(\mathbf{x})|\right.
$$

The posterior energy function is established using the definitions of the Bayes theorem which combine the prior and the likelihood energy terms defined in Eqs. (3) and (10) as follows:

$$
U(\omega \mid \mathbf{x})=U(\omega)+U(\mathbf{x} \mid \omega)
$$

Hence the minimum of the posterior energy provides the optimum SRM $\omega \in C$, which is the maximum a posterior probability solution for the SRM problem (Geman and Geman, 1984). To control and balance the spectral and the contextual information flow during the SRM generation a parameter called "the smoothness parameter" $0 \leq \lambda<1$ is introduced to the posterior energy function in Eq. (11). Hence the full form of the posterior energy function can be written as:

$$
U(\omega \mid \mathbf{x})=\lambda U(\omega)+(1-\lambda) U(\mathbf{x} \mid \omega)
$$

The MAP estimation of the posterior energy in Eq. (12) is determined by using the stochastic Simulated Annealing technique (SA). This implements a Metropolis-Hasting sampling technique to reduce the energy to yield the maximum a posterior solution. A comprehensive explanation about the SA technique can be found in literature ( $\mathrm{Li}, 2009$; Tso and Mather, 2009).

\section{STUDY AREA AND THE DATA PREPERATION}

The fuzzy MRF based SRM technique was tested using real satellite images. Study area was selected in the southwest urban coastal zone of Sri Lanka. In particular this area is undergoing a major construction of a highway running from the south to the west. Three main land cover classes were considered for the 
study, Vegetation (V), Impervious surface (I) and Soil (S). This selection was influenced by the Ridd`s (Ridd, 1995) V-I-S model for the urban composition. Fig. 1 provides the details of the study area, with the Google map images showing the land cover changes during the year 2007 to 2009.
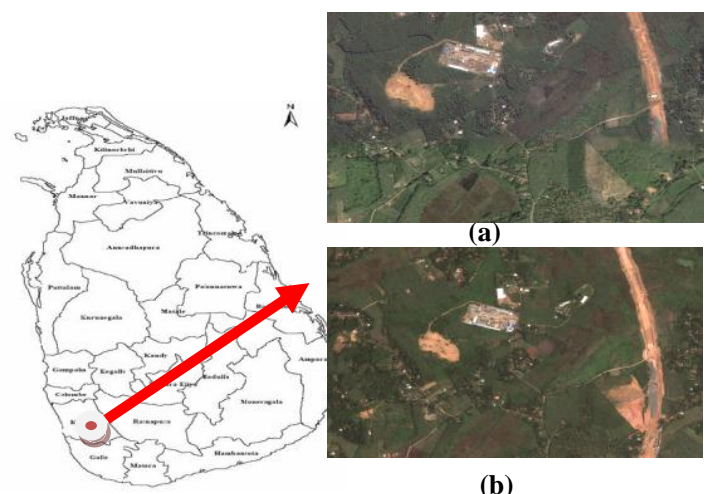

(b)

Fig.1. Google map image of the Study site in the west coast of Sri Lanka, for (a) year 2007 and (b) year 2009
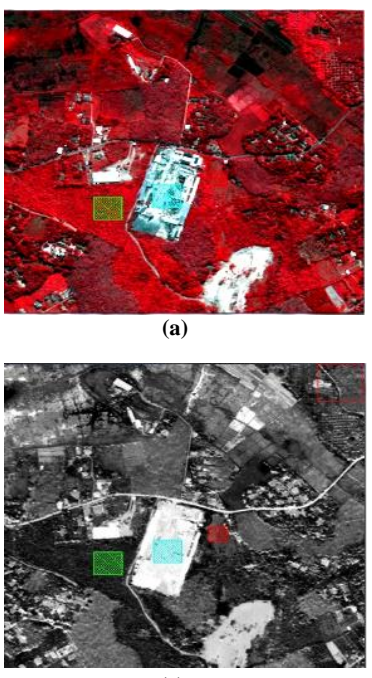

(c)
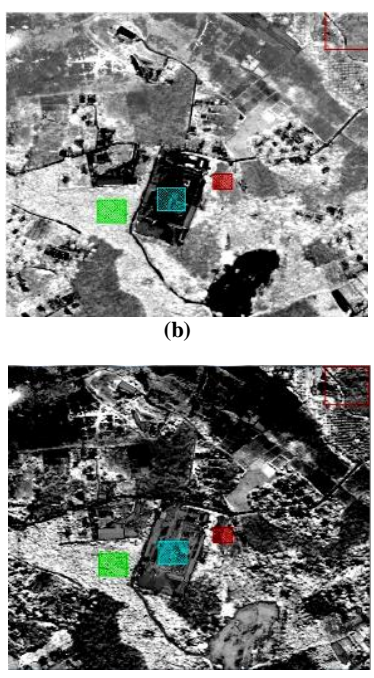

(d)
Vegetation Impervious

Soil

Fig.2. (a) Worldview-II false colour multispectral band composite RGB $(6,5,4)$ with ground samples, (b), (c) and (d) fractional images generated by SAM, for V-I-S classes

A subset image from the DigitalGlobe's WorldView-2 satellite acquired on $29^{\text {th }}$ January 2010 was employed in this study (DigitalGlobe, 2011). The image was preliminary processed to identify the fractional abundance of the V-I-S classes in each pixel. Here the Minimum Noise Fraction (MNF) transformation was employed to remove the correlation exist between the eight bands and to identify the pure pixels in the image. These pure pixels were then plotted on the n-Dimensional (n-D) feature space to further identify the ideal pure signature for each landcover classes of interest. The Spectral Angle Mapper (SAM) technique was performed on the data to generate the fractional images to define the fuzzy membership values for each pixel. The use of SAM in this study was encouraged due to several important reasons, SAM does not necessarily effective to illumination and the albedo effects, image was not corrected for the atmospheric effects and the pixel spectra of the selected classes were well distributed with Transform Divergence (TD) and Jeffries-Matusita distance (JM) being very close to 1.9 and 2.0 for the randomly test samples over the study area. The ability of the SAM to withstand different atmospheric and topographic variation is because that these effects linearly scale the spectra of the unclassified pixel vector and the vectors of the reference signatures belonging to same class, with least effect on the spectral angle. (Sohn et al., 1999). The generated fractional images are considered to be the Fuzzy functional images in this study. Fig.2 shows the results of the SAM classification and the square homogeneous training samples chosen for the three classes to determine the fuzzy class parameters. The square form of the training samples was to ease the calculation process. For vegetation and the impervious surface the total number of training pixels was 900 while for the soil class it was selected to be 400 . Initial parameter determination was performed on a $400 \times 400$ pixel image subset, while for the MRF based SRM input it was further reduced to a $100 \times 100$ pixel image. This is mainly to save the time taken for the processing.

The statistical parameters mean and covariance, generated from the conventional and the fuzzy algorithms are different. The fuzzy covariance matrix for the vegetation class and the conventional and fuzzy means for the three classes V-I-S, are shown in Table 1and Table 2 respectively. The bench mark reference images were produces by performing the hard Maximum Likelihood classification (MLC), using the same training samples implemented for the class parameter estimations. Due to the SRM resolution being $1 \mathrm{~m}$ according to a scale factor of 2, prior to the MLC classification the images were resampled to $1 \mathrm{~m}$ resolution for a pixel to pixel comparison. Considering the distribution of the classes in the study region, to avoid the original pixels being over resampled, nearest neighbour resampling technique has been employed. For the determination of the class composition within a pixel to generate the initial SRM maps, we used the Singular Value Decomposition (SVD) technique (Canty, 2010). In SVD a library matrix determined by the class mean vector is inverted by decomposing it to two column orthogonal matrices and a diagonal matrix to perform a vector multiplication with the observed image to get the least square estimate of end member abundance. If the fractional estimation for a particular class in a pixel $x_{i} \in A$ in the observed image is $\theta_{i}$, then there will be $\theta_{i} \times S^{2}$ allocation of that class in the corresponding pixel set $B$ of the SRM. Figs. 4(a) and 5(a) shows the initial SRM`s generated from the SVD fractions.

\section{EXPERIMENTAL RESULTS}

Experimental results of the MRF based SRM with respect to several smoothness parameters $(\lambda)$ using the fuzzy and the conventional class parameters are shown in the Figs. 3 and 4 respectively. It should be mentioned that the $\lambda$ is a key parameter which controls the contribution of the prior and the likelihood energy in the posterior energy determination. When $\lambda=1$ the likelihood term is completely ignored in Eq. (12) for a minimal posterior energy, which forces all the pixels to be classified to a single class. These figures represents the initial SRM and the Optimized SRM`s for $\lambda=0.7,0.8,0.9$, and the reference image. The validation of the SRM can be done at both pixel level as well as the sub pixel level. In both the cases it is a complex process. In the pixel based validation, we used the Cohen`s kappa statistics (Congalton, 1991), which is also known as kappa coefficient to assess the accuracy of the generated SRM using the contingency tables. For the sub pixel based validation we used the mean absolute error (MAE) (Tang et al., 2007)- 
ISPRS Annals of the Photogrammetry, Remote Sensing and Spatial Information Sciences, Volume I-7, 2012

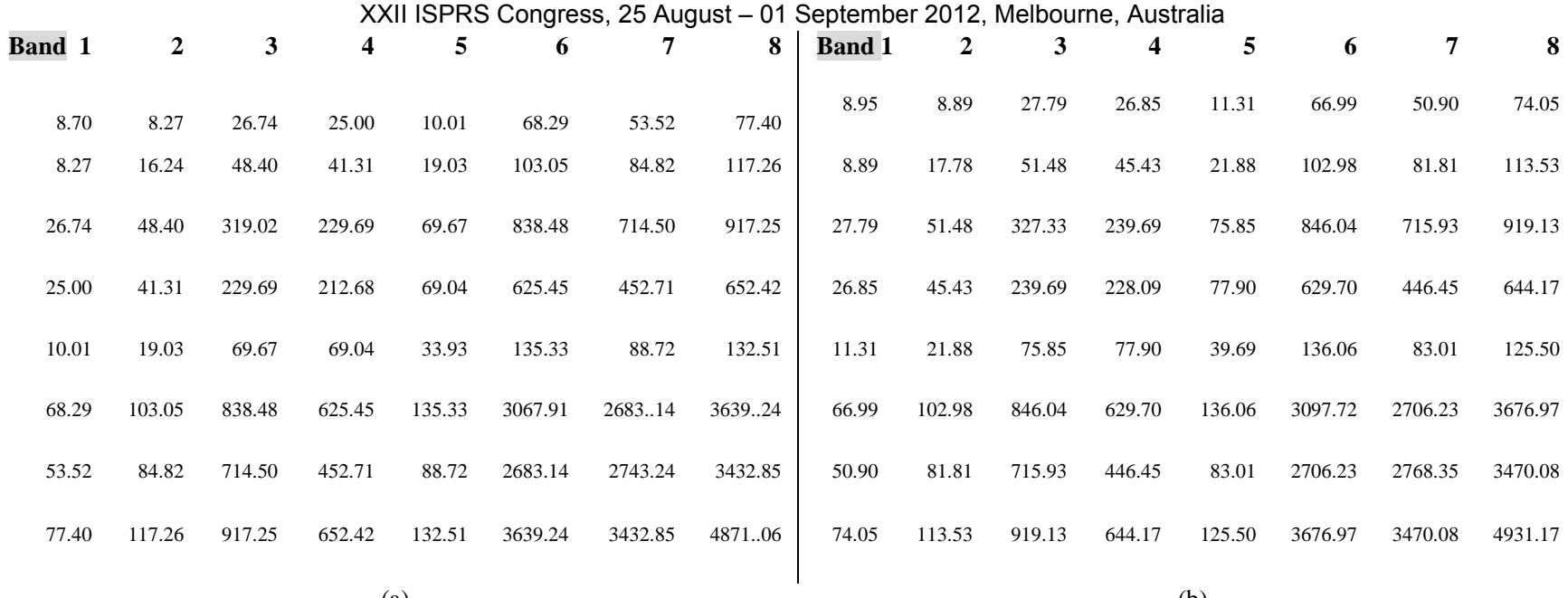

(a)

(b)

Table 1. (a) Fuzzy covariance matrix for the vegetation class and (b) Conventional Covariance matrix for the vegetation class

\begin{tabular}{|c|c|c|c|c|c|c|c|c|c|}
\hline Band & & 1 & 2 & 3 & 4 & 5 & 6 \\
\hline \multirow{3}{*}{ Vegetation } & Fuzzy & 362.99 & 274.47 & 355.50 & 242.71 & 79.46 & 553.95 & 510.57 & 715.65 \\
& Conventional & 363.02 & 274.57 & 355.46 & 242.97 & 79.66 & 552.27 & 508.14 & 712.30 \\
\hline \multirow{2}{*}{ Impervious } & Fuzzy & 478.60 & 443.49 & 617.311 & 581.744 & 261.25 & 515.35 & 308.05 & 411.22 \\
\cline { 2 - 9 } & Conventional & 476.98 & 441.38 & 614.40 & 579.49 & 260.42 & 516.58 & 310.50 & 415.67 \\
\hline \multirow{2}{*}{ Soil } & Fuzzy & 378.34 & 299.54 & 378.28 & 294.68 & 115.59 & 504.69 & 432.93 & 634.73 \\
\cline { 2 - 9 } & Conventional & 378.33 & 299.36 & 377.15 & 293.87 & 115.28 & 499.10 & 426.93 & 625.40 \\
\hline
\end{tabular}

Table 2. Fuzzy and conventional mean values for the V-I-S classes.

-to-compare the class area proportion within a fixed area size, between the SRM and the reference images. The MAE is defined as follows:

$$
M A E_{l}=\frac{\sum_{m=1}^{N} \sum_{l=1}^{l}\left|S R M \quad f r_{l}(m)-{ }^{\mathrm{Re} f} f r_{l}(m)\right|}{N}
$$

Where $N$ is the total number of pixels considered. The ${ }^{S R M} f r_{l}(m)$ and ${ }^{\operatorname{Re} f} f r_{l}(m)$ are the fractional value of class $l$ in a fixed area $m$ of the SRM and the reference image respectively. MAE provides an average measure of the agreement of the class fractions that has changed within a fixed area from the ground truth to thematic output. Hence a small error clearly leads to a better agreement between the two inputs. According to the previous experiments done on the parameters of the MRF technique it has been observed that for smaller scale factors $S \leq 4, \lambda$ takes the values in the range of 0.7 to 0.9 (Tolpekin and Stein, 2009). Confirming these findings in this study for $S=2$, the highest accuracy of the SRM with respect to the reference image was attained at $\lambda=0.9$, for both cases. According to the Table 3 the kappa agreement for the SRM generated at $\lambda=0.9$ for the fuzzy MRF model is 0.78 while its conventional counterpart reached 0.65 accuracy. The overall accuracy has also confirms the agreement with $86 \%$ for the SRM generated by using the fuzzy parameters while $78 \%$ for the conventional parameters. Hence the classification agreement between the optimized SRM and the MLC classified images produced with the fuzzy class parameters are improved with respect to its conventional counterpart. Visual interpretation of the results shows, smoother SRM maps were generated using the fuzzy class parameters. This is presented in the Figs. 3(d) and 4(d). Grass and exposed soil class attains a comparatively higher degree of uncertainty in the parameterization than the other two classes. This is mainly due its higher mixture with the vegetation class.
The joint distribution of normally distributed two variables is expected to have a Gaussian distribution. The determination of the class fractions are from normally distributed pixel vectors of the class samples. These fractions assigned as the membership grades, tend to preserve the original distribution and provide better probabilistic measures for classes within a pixel. This affects the pixel labelling problem with the fuzzy MRF model, demanding the pixels to have accurate statistical measure through the prior and likelihood energy determination than in the conventional method. Hence with the use of the membership grades the classification results has significantly improved for the soil class.

\begin{tabular}{|l|l|l|l|l|l|}
\hline \multicolumn{3}{|c|}{ Fuzzy MRF } & \multicolumn{3}{c|}{ Conventional MRF } \\
\hline $\boldsymbol{\lambda}$ & OA & Kappa & $\boldsymbol{\lambda}$ & OA & Kappa \\
\hline 0.9 & $85.60 \%$ & 0.78 & 0.9 & $77.81 \%$ & 0.65 \\
\hline 0.8 & $84.52 \%$ & 0.76 & 0.8 & $77.26 \%$ & 0.64 \\
\hline 0.7 & $83.04 \%$ & 0.74 & 0.7 & $75.94 \%$ & 0.62 \\
\hline
\end{tabular}

Table 3. Kappa and OA, agreement between the Optimal SRM and the reference image generated using MLC

The persistence of the classification agreement between the ground truth classes and the thematic classes for the vegetation and Impervious categories are slightly higher in the fuzzy parameterized MRF model than its conventional case. The validation was carried out mainly to compare the fuzzy and the conventional MRF results for their accuracy at a pixel level. But it has certain draw backs as the error matrix doesn't provide the convenience to evaluate the effect of the multiple memberships' assignment for each pixel and also the fractional agreement of the fuzzy classification. To evaluate the fractional agreement between the optimal SRM and the reference images, MAE error measure has also been employed. A fixed area size for the comparison was set to $5 \times 5$ pixel area. This fixed area size was- 


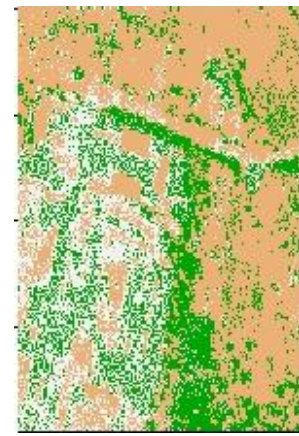

(a)

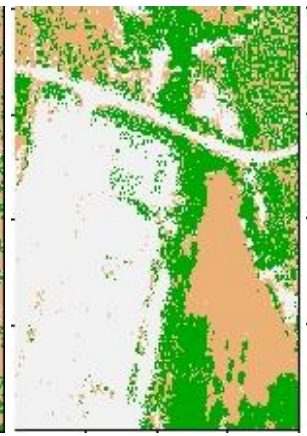

(b) $\lambda=0.7$

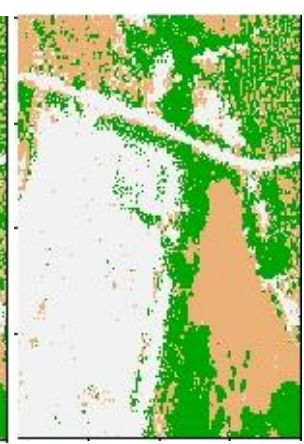

(c) $\lambda=0.8$

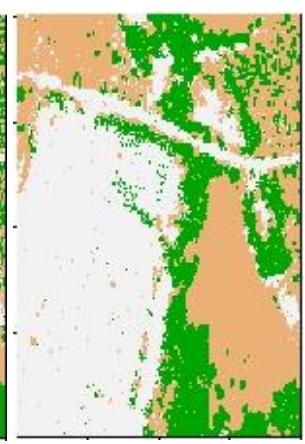

(d) $\lambda=0.9$

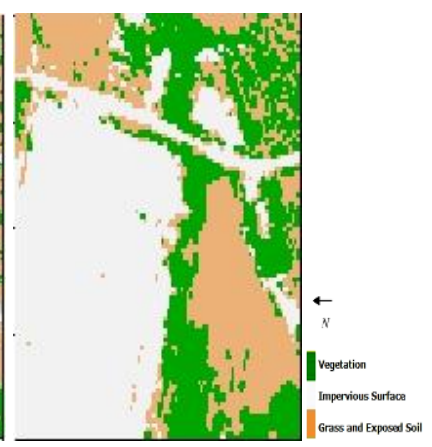

(e)

Fig.3. Initial SRM (a) Optimum SRM generated using fuzzy class definitions for $\lambda=0.7$ (b), $\lambda=0.8(\mathrm{c}), \lambda=0.9$ (d) respectively and the MLC classified reference image (e).

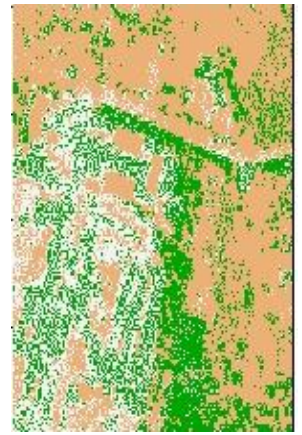

(a)

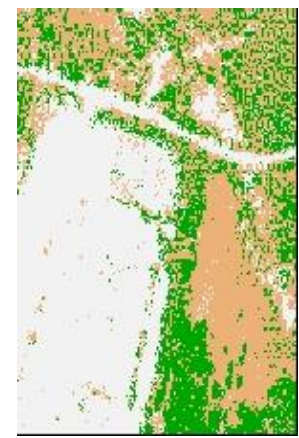

(b) $\lambda=0.7$

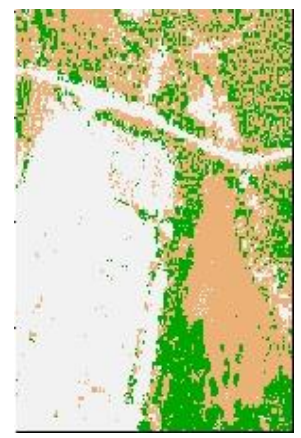

(c) $\lambda=0.8$

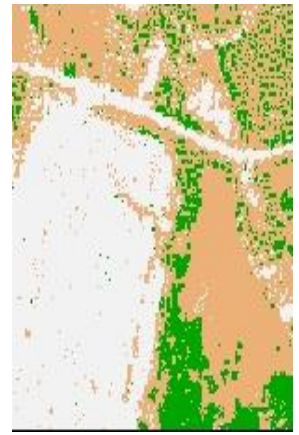

(d) $\lambda=0.9$

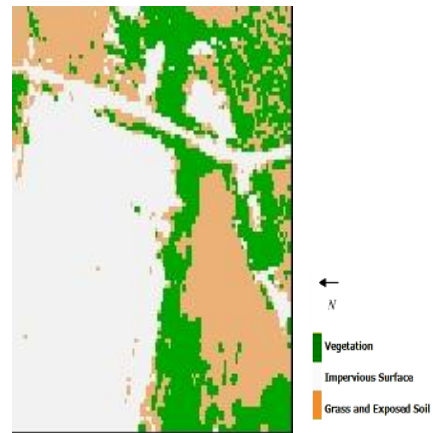

(e)

Fig.4. Initial SRM (a) Optimum SRM generated using conventional class definitions for $\lambda=0.7$ (b), $\lambda=0.8(\mathrm{c}), \lambda=0.9$ (d) respectively and the MLC classified reference image (e).

-chosen so that in this region the MLC classified output provides the necessary mixture condition for all the 3 classes to have fractional estimations. The definition of the fixed area can vary depending on the heterogeneity of the classes in the study region. The proportions of the classes within this fixed area of five by five pixels were determined for both the SRM and the reference images. The MAE for the fuzzy integrated MRF model and the conventional model based SRM are shown in Table 4. Low MAE values with 0.03, 0.02 and 0.05 for the V-I$\mathrm{S}$ classes were resulted for the fractions in the fuzzy integrated MRF model. Both the pixel and sub-pixel level evaluation of the results with respect to the MLC based reference data; suggest that the use of fuzzy class definitions provides more promising results than in the conventional case. It is also more effective for the ambiguous classes for ex. Soil.

\begin{tabular}{|c|l|c|c|c|c|}
\hline \multirow{4}{*}{ MAE } & Vegetation & Soil & Impervious & $\begin{array}{c}\text { Average } \\
\text { MAE }\end{array}$ \\
\cline { 2 - 6 } & Fuzzy MRF & 0.03 & 0.05 & 0.02 & 0.03 \\
\cline { 2 - 6 } & $\begin{array}{l}\text { Conventional } \\
\text { MRF }\end{array}$ & 0.12 & 0.15 & 0.03 & 0.10 \\
\hline
\end{tabular}

Table 4. Comparison of the mean absolute error (MAE) for the fuzzy integrated MRF model and the conventional MRF model.

\section{CONCLUSIONS AND DISCUSSION}

This work investigate the improvements of the MRF based SRM technique with the integration of the fuzzy class parameters. The testing was done using a Worldview-II data set over a semi urban environment. The main assumptions made in the study were the selected training pixels to be pure elements of their representative land cover classes, the fine resolution pixels (y) are conditionally independent, fuzzy membership grade of a pixel (x) for a particular class is its proportion within that pixel and the observed pixel vector $x_{m}$ of the multispectral image is having a Gaussian distribution with mean $\bar{\mu}(\mathrm{x})$ and covariance $\bar{\Sigma}(\mathrm{x})$ (Eqs.(7) and (8)) defined using fuzzy definitions. The healthy class spectral seperability with TD and JM close to 1.9 and 2.0 respectively suggested the least spectral confusion among the classes in the study area. This improves the selection of the pure spectral elements or the endmembers to perform the SAM classification with good accuracy. Minimization of the posterior energy for the optimum SRM $c$, (Eq. (12)) was achieved using SA with Metropolis-Hasting sampler (Geman and Geman, 1984). The main parameter which controls the annealing schedule of SA technique is called the initial temperature $\left(T_{0}\right)$ and the cooling schedule parameter $(\kappa)$ which control the rate of temperature decrease (Li, 2009). Both these parameters are determined on the basis of the complexity of the problem at hand (Tolpekin and Stein, 2009). With the $S=2$ and the higher class spectral seperability the complexity of the classification problem addressed in this study is considerably low. For better results in a complex situations, large values of both these parameters $\left(T_{0}=3.0\right.$ and $\left.\kappa=0.9\right)$ which slows down the annealing process is recommended (Tolpekin and Stein, 2009). Regardless of the low complexity of the classification problem in this study, we have implemented a slow annealing process for better results as a precaution. We tested the fuzzy and conventional MRF models for a range of $\lambda$ 
values from 0.3 to 0.9 . SRM generated for each of these $\lambda$ parameters is a configuration of different spectral and contextual information combination. As a benchmark these SRM results were comprehensively evaluated with respect to a standard parametric classification technique (MLC) at both pixel and sub pixel levels. For both the cases at a $\lambda=0.9$ the optimum SRM were generated. The results demonstrate the significant improvements in the fuzzy MRF model, with smoother classification results.

The overall results suggested that the use of SAM for the generation of fuzzy functional images is reasonable. Using membership grades for the training pixels is observed to be advantageous as it also reduce the amount of representative training samples needed for the classification. An extension is needed to investigate into the different optimal fuzzy membership definition methods. The contextual information integration and the improvement of the class parameters using fuzzy definitions in the MRF based SRM technique, is proved to be more effective with better classification accuracy. For vague land cover interpretations this approach can be very advantages due to its capabilities to reach higher classification accuracies at the sub pixel level. Further work is needed to test the performance of the method at a coarser resolution scale with heterogeneous land cover classes with strong similarity and also to validate the results with reference images having higher accuracy at sub pixel levels.

\section{ACKNOWLEDGEMENT}

This research was supported by Kyoto University Global COE Program "Global Centre for Education and Research on Human Security Engineering for Asian Megacities". The authors would also like to thank the Faculty of Geomatics, Sabaragamuwa University of Sri Lanka for providing the Remote Sensing data.

\section{REFRENCES}

Ardila, J.P., Tolpekin, V. A, Bijker, W., Stein, A., 2011. Markov-random-field-based super-resolution mapping for identification of urban trees in VHR images. ISPRS Journal of Photogrammetry and Remote Sensing 66 (6), 762-775.

Canty, M. J., 2010. Image Classification, and Change Detection in Remote Sensing with algorithms for ENVI/IDL. CRC Press, Boca Raton.

Congalton, R. G., 1991. A review of the assessing the accuracy of classification of Remotely Sensed data. Remote Sensing of Environment 37 (1), 35-46.

DigitalGlobe., 2011. www.digitalglobe.com (Accessed 20 February, 2011)

Fisher, P., Arnot, C., Wadsworth, R., Wellens, J., 2006. Detecting change in vague interpretations of Landscapes. Ecological Informatics 1 (2), 163-178.

Geman, S., Geman, D., 1984. Stochastic relaxation, Gibbs distribution, and the Bayesian restoration of images. IEEE Transactions on Pattern Analysis and Machine Intelligence 6 (6), 721-741.

Kasetkasem, T., Arora, M. K., Varshney, P. K., 2005. Superresolution land cover mapping using a Markov Random Field based approach. Remote Sensing of Environment 96 (3-4), 302-314.

Kasetkasem, T., Varshney, P. K., 2002. An Image Change Detection Algorithm based on Markov Random Field
Model. IEEE Transactions on Geoscience and Remote Sensing 40(8), 1815-1823.

Li, S. Z., 2009. Markov Random Field Modelling in Image Analysis, thired ed, Springer-Verlag, London.

Pal, M., Mather, P. M., 2004. Assessment of the Effectiveness of Support Vector Machines for Hyperspectral data. Future Generation Computer Systems 20 (7), 12151225.

Richards, J. A., Jia, X., 2006. Remote Sensing Digital Image Analysis, fourth ed. Springer-Verlag.

Ridd, M. K., 1995. Exploring a V-I-S(Vegetation-impervious surface-soil) Model for Urban Ecosystem Analysis through Remote Sensing: comparative anatomy for cities. International Journal of Remote Sensing 16(12), 2165-2185.

Sohn, Y., Moran, E., Gurri, F., 1999. Deforestation in NorthCentral Yucatan (1985-1995): Mapping secondary succession of forest and agricultural land use in sotuta using the cosine of the angle concept. Photogrammetric Engineering and Remote Sensing 65(8), 947-958.

Solberg , A. H. S., Taxt, T., Jain, A. K., 1996. A Markov Random Field model for classification of multisource satellite imagery. IEEE Transactions on Geoscience and Remote Sensing 34(1), 100-113.

Tang, J., Wang, L., Myint, S. W., 2007. Improving urban classification through fuzzy supervised classification and spectral mixture analysis. International Journal of Remote Sensing 28(18), 4047-4063.

Tolpekin, V. A., Stein, A., 2009. Quantification of the effects of land-cover-class spectral separability on the accuracy of Markov-Random-Field-Based Superresolution Mapping. IEEE Transactions on Geoscience and Remote Sensing 47(9): 3283-3297.

Tso, B., Mather, P. M., 2009. Classification Methods for Remotely Sensed data, second ed. CRC Press, Boca Raton.

Wang, F., 1990. Fuzzy Supervised Classification of Remote Sensing Images. IEEE Transactions on Geoscience and Remote Sensing 28(2), 194-201.

Welikanna, D. R., Tolpekin, V. A., Kant, Y., 2008. Analysis of the effectiveness of spectral mixture analysis and the Markov Random Field based Super Resolution Mapping over an Urban Environment, International Archives of Photogrammetry, Remote Sensing and Spatial Information Sciences 37 (part B7), 641-650.

Wood, T. F., Foody, G. M., 1993. Using cover-type likelihoods and typicalities in a Geographic Information System data structure to map gradually changing environments, Landscape Ecology and GIS 141-146.

Xu, M., Chen, H., Varshney, P. K., 2011. An image fusion approach based on Markov Random Fields. IEEE Transactions on Geoscience and Remote Sensing 49(12), 5116-5127.

Zadeh, L. A., 1965. Fuzzy Sets. Information and Control 8 (3), 338-353.

Zhang, J., Foody, G. M., 2001. Fully-fuzzy supervised classification of sub-urban land cover from remotely sensed imagery: statistical and artificial neural network approach. International Journal of Remote Sensing, 22 (4), 615-628. 